USTC-ICTS-09-13

\title{
Holographic Superconductor for a Lifshitz fixed point
}

\author{
Sang-Jin Sin!, Shan-Shan Xu², Yang Zhou芆 \\ 1)Department of Physics, Hanyang University, Seoul 133-791, Korea \\ 2)Interdisciplinary Center for Theoretical Study \\ University of Science and Technology of China, Hefei, Anhui 230026, China \\ 3)Institute of Theoretical Physics, Chinese Academic of Science, Beijing 100190, PRC \\ Kavli Institute for Theoretical Physics China at the Chinese Academy of \\ Sciences(KITPC-CAS)
}

\begin{abstract}
We consider the gravity dual of strongly coupled system at a Lifshitz-fixed point and finite temperature, which was constructed in a recent work arXiv:0909.0263. We construct an Abelian Higgs model in that background and calculate condensation and conductivity using holographic techniques. We find that condensation happens and DC conductivity blows up when temperature turns below a critical value.
\end{abstract}

\footnotetext{
${ }^{1}$ E-mail:sangjin.sin@gmail.com

${ }^{2}$ E-mail: xuss@mail.ustc.edu.cn

${ }^{3}$ E-mail:yzhou@itp.ac.cn
} 


\section{Contents}

1 Introduction $\quad 2$

2 Gravity dual of the Lifshitz fixed point 3

2.1 Lifshitz black hole solutions . . . . . . . . . . . . . . . . . . . 3

2.2 Thermodynamics ..................... 4

2.3 Finite chemical potential . . . . . . . . . . . . . 6

3 Superconductivity

3.1 Superconductive phases . . . . . . . . . . . . . . . . . . . . 7

3.2 Conductivity . . . . . . . . . . . . . . . . . . . . . 10

\section{Introduction}

AdS/CFT correspondence [1] is one of the most interesting results in the sense that it opened a window of connecting the string theory to the QCD and condensed matter systems. The connection between gauge theory and strings has a long history since the appearance of string models of hadrons in 1960's: For example, it has been observed that the elementary excitations of a lattice gauge theory in the strong coupling limit can be represented by strings formed by color-electric fluxes. It is also suggested that in a certain limit all the degrees of freedom in the gauge theory should be represented by the flux lines (strings) instead of fields. See [3], [2] and references therein. Therefore it is natural to expect an exact duality between gauge fields and strings although its precise formulation was obtained only recently. The semi-classic version of this duality can be stated as gauge/gravity duality and such duality has become a powerful tool to understand the strongly coupled QCD and the properties of quark gluon plasma in heavy ion collisions at RHIC [4, 5, 6] as well as the low energy hadron physics.

More recently, it has been attempted to use this correspondence to describe certain condensed matter systems such as the Quantum Hall effect [7], Nernst effect [8, 9, 10], superconductor [11, 13, 14] and fractional quantum hall effect (FQHE) [15]. These phenomena were suggested to have dual gravitational descriptions. As pointed out in [16], there is a large class of interesting strongly correlated electronic and atomic systems that can be created and studied in experiments and there are non-relativistic systems which have Schrödinger symmetry [16]. However, the dynamics of such systems near a critical 
point is described by a relativistic conformal field theory or sometimes more subtle scaling theory having Lifshitz symmetry [17].

To describe the finite temperature version of such scaling system, four dimensional black hole solutions with asymptotically Lifshitz spacetimes were investigated [18, 19, 20, 21, 22. The Lifshitz black holes in arbitrary dimensions were also found in a different class of action [23]. Recently an analytical solution in yet another action was proposed for $\mathrm{z}=2$ [24] in four dimension. Additionally, Lifshitz black holes in three-dimensional massive gravity and four-dimensional $R^{2}$ gravity were also discussed [25, 26]. Embedding those black holes with the action in [17] into string theory was addressed in [27].

In [11, a model of a strongly coupled system which shows superconductivity was constructed based on holography, which is an Abelian-Higgs model in a warped space time. While the electrons in real materials are non-relativistic, the model in [11] is relativistic. Therefore it is natural to ask whether one can develop a similar model with non-relativistic kinematics [34], especially at Lifshitz-like fixed point. One purpose of this paper is to answer this question. We find that there is a critical temperature, like the relativistic case, below which a charged scalar field condensate and the (DC) conductivity blows up. We also calculated the frequency dependent conductivity.

This paper is organized as follows. In section 2, we check thermodynamics of the Lifshitz black hole and chemical potential background. In the following section, we study the superconductive phases in the Lifshitz background. We obtain similar results as in the usual AdS black hole background.

\section{Gravity dual of the Lifshitz fixed point}

We begin with the equilibrium properties of the strongly coupled thermal field at Lifshitzlike fixed point, by analyzing the Lifshitz black hole solutions.

\subsection{Lifshitz black hole solutions}

The Lifshitz scaling is defined by

$$
t \rightarrow \lambda^{z} t, \quad x \rightarrow \lambda x
$$

where $z$ is called dynamical exponent. The metric with this symmetry was first found in [17]:

$$
d s^{2}=L^{2}\left(-\frac{d t^{2}}{r^{2 z}}+\frac{d x^{2}+d y^{2}}{r^{2}}+\frac{d r^{2}}{r^{2}}\right),
$$


where $0<r<\infty$ and $L$ sets the scale for the radius of curvature. For $z=1$, this geometry is anti-de sitter spacetime. For $z>1$, it is a candidate for the dual gravity of a field theory with Lifshitz scaling.

The tidal forces diverge on the "horizon" at $r \rightarrow \infty$ unless $z=1$ and this implies that the metric (2) has no global extension [28]. To describe the physics of the dual field theory at finite temperature, black hole solutions with asymptotical Lifshitz metric (2) were studied in [18, 19, 20, 21, 22].

Recently, a 4D black hole solution which asymptotes to the Lifshitz spacetime (2) was constructed[24]. The action is

$$
S=\frac{1}{2} \int d^{4} x \sqrt{-g}(R-2 \Lambda)-\int d^{4} x \sqrt{-g}\left(\frac{e^{-2 \phi}}{4} F_{\mu \nu} F^{\mu \nu}+\frac{m^{2}}{2} A_{\mu} A^{\mu}+\left(e^{-2 \phi}-1\right)\right)
$$

where $\Lambda=-\frac{z^{2}+z+4}{2}, m^{2}=2 z$ and $F=d A$. The gravitational constant and curvature radius are set by $8 \pi G_{4}=1$ and $L=1$ respectively. With this convention the equations of motion are

$$
\begin{aligned}
F^{2} & =-4, \quad \frac{1}{\sqrt{-g}} \partial_{\mu}\left(\sqrt{-g} e^{-2 \phi} F^{\mu \nu}\right)=m^{2} A^{\nu} \\
R_{\mu \nu} & =e^{-2 \phi} F_{\mu \lambda} F_{\nu}^{\lambda}+m^{2} A_{\mu} A_{\nu}+\Lambda g_{\mu \nu}+\left(2 e^{-2 \phi}-1\right) g_{\mu \nu}
\end{aligned}
$$

and the black hole solution of this system is 4

$$
\begin{array}{r}
d s^{2}=-f(r) \frac{d t^{2}}{r^{2 z}}+\frac{d x^{2}+d y^{2}}{r^{2}}+\frac{d r^{2}}{r^{2} f(r)}, \\
f(r)=1-\frac{r^{2}}{r_{H}^{2}}, \quad e^{-2 \phi}=1+\frac{r^{2}}{r_{H}^{2}}, \quad A=\frac{f(r)}{\sqrt{2} r^{2}} d t .
\end{array}
$$

In the rest of this paper, we will use this solution to discuss the transport and superconductivity.

\subsection{Thermodynamics}

We first review the thermodynamics of this black hole proposed in [24]. Our calculational procedure follows [28]. According to the AdS/CFT dictionary, the partition function of the bulk theory is identified to that of the dual field theory. The path integral over metrics is dominated by the saddle point $g_{*}$, and the partition function is

$$
Z=e^{-S_{E}\left[g_{*}\right]}
$$

\footnotetext{
${ }^{4}$ There is a factor $1 / \sqrt{2}$ missing in the expression (2.5) of the massive vector field in [24].
} 
where $S_{E}\left[g_{*}\right]$ is the Euclidean action evaluated on the saddle. This action must contain extrinsic boundary terms and intrinsic boundary terms in order to render the finiteness of the on-shell action. This was already given in [24]:

$$
\begin{aligned}
S_{E}= & -\frac{1}{2} \int d^{4} x \sqrt{g}(R-2 \Lambda)+\int d^{4} x \sqrt{g}\left(\frac{e^{-2 \phi}}{4} F^{2}+\frac{m^{2}}{2} A^{2}+\left(e^{-2 \phi}-1\right)\right) \\
& +\int_{r \rightarrow 0} d^{3} x \sqrt{\gamma} K-\frac{1}{2} \int_{r \rightarrow 0} d^{3} x \sqrt{\gamma}\left(-\frac{27}{8}+\frac{7}{2} \phi+\frac{7}{2} \phi^{2}\right) \\
& -\frac{1}{2} \int_{r \rightarrow 0} d^{3} x \sqrt{\gamma}\left(\left(\frac{17}{2}+7 \phi\right) A^{2}+\frac{13}{2} A^{4}\right)
\end{aligned}
$$

where $\gamma$ is the induced metric on the boundary $r \rightarrow 0$ and $K$ is the trace of the extrinsic curvature. The Dirichlet boundary condition is imposed on the massive vector field 5

A saddle is obtained by Wick rotating eq. (5):

$$
d s_{*}^{2}=f(r) \frac{d \tau^{2}}{r^{2 z}}+\frac{d x^{2}+d y^{2}}{r^{2}}+\frac{d r^{2}}{r^{2} f(r)}, \quad A=-i \frac{f(r)}{\sqrt{2} r^{2}} d \tau .
$$

The temperature of the system is

$$
T=\frac{1}{\beta}=\frac{1}{2 \pi r_{H}^{z}}
$$

determined by the absence of the conical singularity at $r=r_{H}$.

We can now evaluate the action (7):

$$
S_{E}\left[g_{*}\right]=-\beta \frac{L_{x} L_{y}}{2 r_{H}^{4}}=-2 \pi^{2} L_{x} L_{y} T
$$

and the free energy

$$
\mathcal{F}=-T \log Z=T S_{E}\left[g_{*}\right]=-\frac{L_{x} L_{y}}{2 r_{H}^{4}}=-2 \pi^{2} L_{x} L_{y} T^{2},
$$

as given in [24].

As a check, the entropy

$$
\mathcal{S}=-\frac{\partial \mathcal{F}}{\partial T}=4 \pi^{2} L_{x} L_{y} T
$$

coincides with the Bekenstein-Hawking entropy $\mathcal{S}=2 \pi A$ with the area of the event horizon $A=L_{x} L_{y} / r_{H}^{2}$ and the unit convention $8 \pi G=1$.

\footnotetext{
${ }^{5}$ That is $c_{N}=0$ in the expression (3.3) in 24]. We rewrote that expression into Euclidean space and substitute the specific values of $c_{0}-c_{5}$. There is a minus sign difference of $c_{1}-c_{5}$ here from those given in Appendix A of [24.
} 
The boundary stress tensor resulting from (7) is

$$
\begin{aligned}
T_{\mu \nu} \equiv & -\frac{2}{\sqrt{-\gamma}} \frac{\delta S}{\delta \gamma^{\mu \nu}}=K_{\mu \nu}-\left(\frac{17}{2}+7 \phi+13 A^{2}\right) A_{\mu} A_{\nu}-K \gamma_{\mu \nu} \\
& +\frac{1}{2}\left(-\frac{27}{8}+\frac{7}{2} \phi+\frac{7}{2} \phi^{2}\right) \gamma_{\mu \nu}+\frac{1}{2}\left(\left(\frac{17}{2}+7 \phi\right) A^{2}+\frac{13}{2} A^{4}\right) \gamma_{\mu \nu},
\end{aligned}
$$

then the internal energy and pressure of boundary theory following [24] are respectively

$$
\begin{aligned}
\mathcal{E} & =-L_{x} L_{y} \sqrt{-\gamma} T_{t}^{t}=\frac{L_{x} L_{y}}{2 r_{H}^{4}}, \\
\mathcal{P} & =\frac{1}{2} L_{x} L_{y} \sqrt{-\gamma} T_{i}^{i}=L_{x} L_{y} \sqrt{-\gamma} T_{x}^{x}=\frac{L_{x} L_{y}}{2 r_{H}^{4}} .
\end{aligned}
$$

Thus we have

$$
\mathcal{E}=\mathcal{P}=-\mathcal{F}=\frac{1}{2} T \mathcal{S}
$$

The first law of thermodynamics $\mathcal{E}+\mathcal{P}=T \mathcal{S}$ is satisfied as given in [24].

\subsection{Finite chemical potential}

Now we consider a probe gauge field fluctuation $A_{\mu}$ in the Lifshitz black hole background. That means we need to add another Maxwell term to the original action (3) 77

$$
S=-\frac{1}{4} \int d^{4} x \sqrt{-g} F_{\mu \nu} F^{\mu \nu} .
$$

For simplicity, we ignore the back reaction. It means that $A_{\mu}$ is a small perturbation and the metric is still the same as (5). This vector field is expected to support a charge current operator $J_{\mu}$ in the dual field. The equation of motion of $A_{\mu}$ is

$$
\frac{1}{\sqrt{-g}} \partial_{\mu}\left(\sqrt{-g} F^{\mu \nu}\right)=0
$$

If we only consider the zero component of $A_{\mu}, A=\phi(r) d t$, then we have

$$
\phi^{\prime \prime}+\frac{z-1}{r} \phi^{\prime}=0 \text {. }
$$

Near the boundary,

$$
\phi=\phi_{(0)}+\phi_{(1)} r^{2-z}
$$

\footnotetext{
${ }^{6}$ There are also some minus sign differences between the expression we give and the one in (3.4) of 24.

${ }^{7}$ In this subsection and the rest of this paper, $A$ and $F$ should be distinguished with those in the original action (3) where the vector filed is not a gauge field. From now on, by $A_{\mu}$ and $F$ we mean the Maxwell fluctuation and its strength.
} 
where $\phi_{(0)}$ and $\phi_{(1)}$ are chemical potential and charge density respectively in the dual field theory if $z<2$. In the special case $z=2$,

$$
A_{0}=\mu_{0}-\rho \log r / r_{*}
$$

The coefficient $\rho$ of the log term is precisely the charge density, which in grand canonical ensemble, is defined as the derivative of the boundary action term with respect to the chemical potential $\mu_{0}$. See also 35] for related discussion.

\section{Superconductivity}

In this section, we will build an Abelian-Higgs model [30, 11] in the Lifshitz black hole background and study the superconductive phase. We introduce a new $U(1)$ gauge field $A_{\mu}$ which is different from that in the action (6) and also introduce a complex scalar $\psi$. We assume that the background response is negligible for simplicity.

\subsection{Superconductive phases}

Considering the Lagrangian density

$$
\mathcal{L}=-\frac{1}{4} F^{\mu \nu} F_{\mu \nu}-|\nabla \psi-i A \psi|^{2}-V(|\psi|)
$$

we have equations of motion for $A$ and $\psi$ are respectively

$$
\begin{gathered}
\frac{1}{\sqrt{-g}} \partial_{\mu}\left(\sqrt{-g} F^{\mu \nu}\right)=i q\left[\psi^{*}\left(\partial^{\nu}-i q A^{\nu}\right) \psi-\psi\left(\partial^{\nu}+i q A^{\nu}\right) \psi^{*}\right], \\
\frac{1}{\sqrt{-g}} \partial_{\mu}\left(\sqrt{-g}\left(\partial^{\mu} \psi-i q A^{\mu} \psi\right)\right)-i q A^{\mu}\left(\partial_{\mu} \psi-i q A_{\mu} \psi\right)-\frac{\psi}{2|\psi|} V^{\prime}(|\psi|)=0 .
\end{gathered}
$$

We will work in the probe limit, in which $A_{\mu}$ and $\psi$ are taken to be small so that their back reaction on the spacetime metric can be ignored. The metric is still a $4 \mathrm{D}$ Lifshitz black hole with $\mathrm{z}=2$ in (5).

Taking the ansatz $A=\phi(r) d t, \psi=\psi(r)$, these equations of motion reduce to

$$
\begin{gathered}
\phi^{\prime \prime}+\frac{z-1}{r} \phi^{\prime}-\frac{2 \psi^{2}}{r^{2} f(r)} \phi=0, \\
\psi^{\prime \prime}+\left[\frac{f^{\prime}(r)}{f(r)}-\frac{z+1}{r}\right] \psi^{\prime}+\frac{r^{2 z-2} \phi^{2}}{f^{2}(r)} \psi-\frac{V^{\prime}(\psi)}{2 r^{2} f(r)}=0 .
\end{gathered}
$$

where $\psi$ can be taken to be real which is allowed by the r-component of 22 . For 


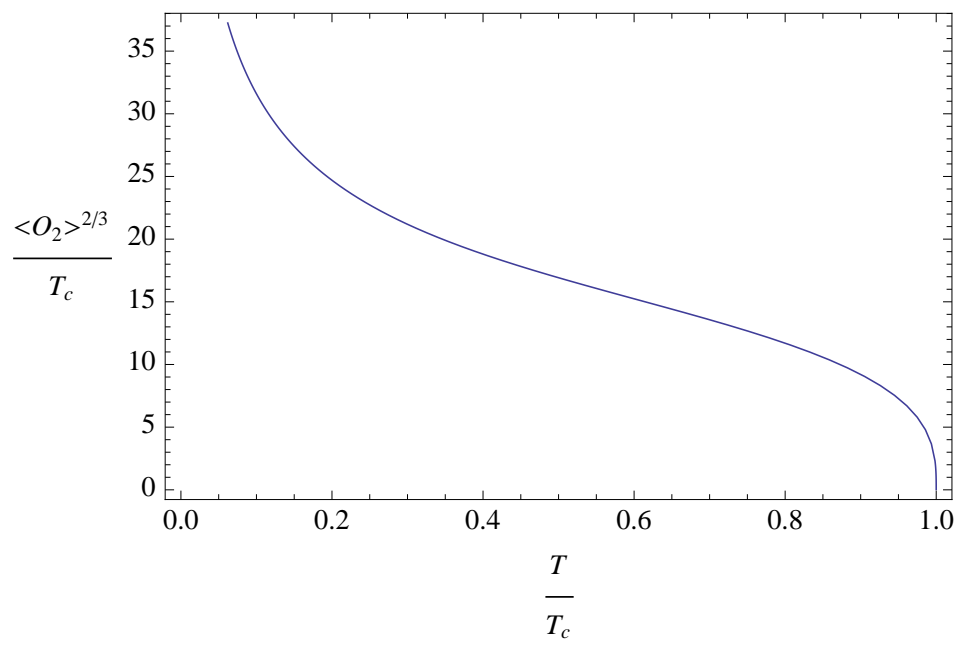

Figure 1: Condensation curve at $z=2, m^{2}=-3 .<O_{2}>=\psi_{(1)}$.

simplicity we will specialize to a simple potential $V(\psi)=m^{2}|\psi|^{2}$ with $m^{2}<0$ but above the Breitenlohner-Freedman bound. Then near the boundary $r \rightarrow 0$ the bulk fields behave as

$$
\begin{gathered}
\phi=\mu+\rho r^{2-z} \ldots \\
\psi=\psi_{(0)} r^{\nu_{-}}+\psi_{(1)} r^{\nu_{+}}+\ldots
\end{gathered}
$$

with $\nu_{ \pm}=\frac{z+2}{2} \pm \sqrt{m^{2}+\left(\frac{z+2}{2}\right)^{2}}$. At the horizon $\phi\left(r_{H}\right)=0$ and 25 implies

$$
\psi^{\prime}\left(r_{H}\right)=-\frac{m^{2}}{2 r_{H}} \psi\left(r_{H}\right)
$$

For $z=2$, as we mentioned before, there is a Log sigularity for the second term in the right hand side of (26). We will study the regularized on-shell action in the following. Including $\phi(r)$ and $\psi(r)$, the bulk action can be rewritten as

$$
S_{b u l k}=V_{3} \int d r \sqrt{-g}\left[-\frac{1}{2} g^{r r} g^{t t}\left(\partial_{r} \phi\right)^{2}-g^{r r}\left(\partial_{r} \psi\right)^{2}-g^{t t} \phi^{2} \psi^{2}-m^{2} \psi^{2}\right]
$$

After doing the above integral by part and using the equations of motion, we have

$$
S_{\text {on-shell }}=V_{3}\left[\left.\sqrt{-g} \phi\left(-\frac{1}{2} g^{r r} g^{t t} \partial_{r} \phi\right)\right|_{r_{B}} ^{r_{H}}+\left.\sqrt{-g} \psi\left(-g^{r r} \partial_{r} \psi\right)\right|_{r_{B}} ^{r_{H}}+\int_{r_{B}}^{r_{H}} \sqrt{-g} g^{t t} \phi^{2} \psi^{2}\right]
$$

Actually, the properties of boundary behaviors of all three terms in (30) heavily depend on the parameter $\nu_{ \pm}$related to $m$. In our discussion, the asymptotical behaviors of 
the second and the third term in (30) are suppressed by $\psi(r)$. Using the asymptotical behaviors of $\phi(r)$ and $\psi(r)$, with eq. 26) replaced by

$$
\phi(r)=\mu_{0}-\rho \log \frac{r}{r_{*}}+\cdots,
$$

theaction can be given by

$$
S=S_{\text {on-shell }}=V_{3}\left[-\frac{1}{2} \rho\left(\mu_{0}-\rho \log \frac{\epsilon}{r_{*}}\right)+\cdots\right],
$$

where $r_{*}$ was introduced in 20 . Finally the regulated Euclidean total action of boundary field theory is given by

$$
S=\frac{V_{2}}{T}\left(-\frac{1}{2} \rho \mu_{s c}+\int_{r_{B}}^{r_{H}} \sqrt{-g} g^{t t} \phi_{c}^{2} \psi_{c}^{2}\right)
$$

where $\mu_{s c}=\mu_{0}$. We need to integrate the classical solution $\phi_{c}$ and $\psi_{c}$. The free energy is obtained by Legendre transformation:

$$
F_{s c}=T S+\mu_{s c} \rho V_{2}=V_{2}\left(\frac{1}{2} \rho \mu_{s c}+\int_{r_{B}}^{r_{H}} \sqrt{-g} g^{t t} \phi_{c}^{2} \psi_{c}^{2}\right) .
$$

For the normal state, the free energy is given by setting $\psi_{c}=0$ in 33

$$
F_{n}=V_{2}\left(\frac{1}{2} \rho \mu_{n}\right)=V_{2}\left(\frac{1}{2} \rho^{2} \log \frac{r_{H}}{r_{*}}\right),
$$

where we use the horizon regularity condition $A_{0}\left(r_{H}\right)=0$ and $\mu_{n}=\rho \log \frac{r_{H}}{r_{*}}$.

In the case with condensation, $\nu_{ \pm}$is simplified to $\nu_{-}=1, \nu_{+}=3$ with $m^{2}=-3$. Note that $\int_{\epsilon}^{r_{H}} \sqrt{-g} g^{t t} \phi_{c}^{2} \psi_{c}^{2}$ has a finite value.

The condensate of the scalar operator $\mathcal{O}$ is encoded in the dual field $\psi$ by

$$
\langle\mathcal{O}\rangle=\psi_{(1)}
$$

with the boundary condition $\psi_{(0)}=0$. We can solve the equations 24 and 25 numerically and finally get a condensation curve shown in figure 1 . Near the critical temperature, this curve is similar to that in BCS theory and that in $\mathrm{z}=1$ holographic superconductor [11]. $\langle\mathcal{O}\rangle$ goes to a finite value as the temperature turns below a critical value. By dimensional analysis, $T_{c} \sim \mu$.

We plot the difference of free energy difference

$$
\Delta F=F_{n}-F_{s c}=V_{2}\left(\frac{1}{2} \rho\left(\mu_{n}-\mu_{s c}\right)+\int_{\epsilon}^{r_{H}} \sqrt{-g} g^{t t} \phi_{c}^{2} \psi_{c}^{2}\right) .
$$

where $\mu_{n}, \mu_{s c}$ mean chemical potential at the normal and superconducting phase respectively. Figure 2 demonstrates that the free energy for superconducting state is lower below critical temperature. 


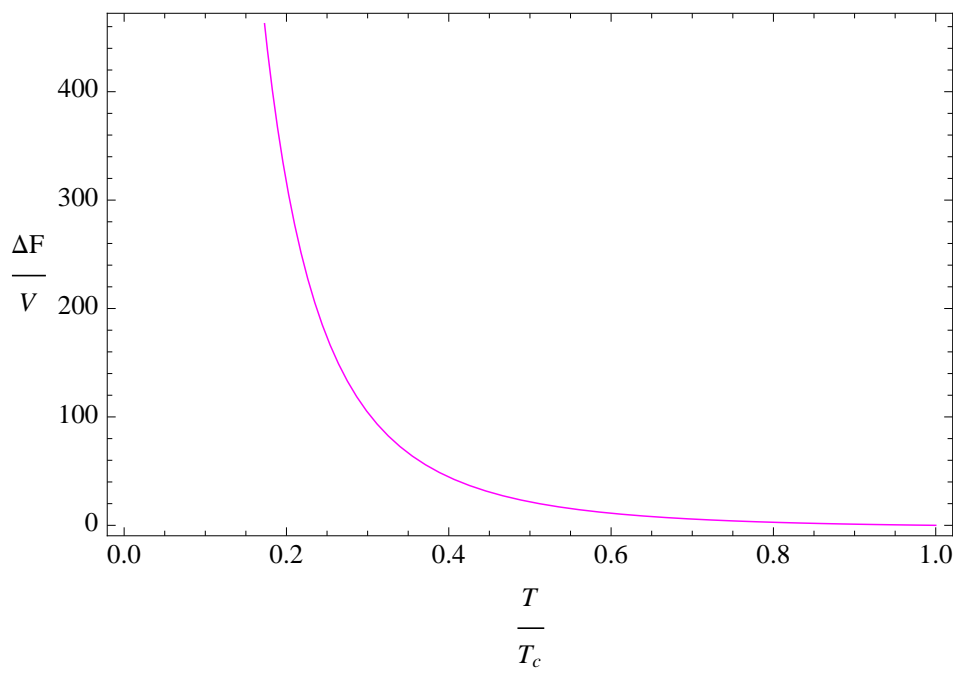

Figure 2: Difference of free energy curve with and without condensation, $\Delta F=F_{n}-F_{s c}$.

\subsection{Conductivity}

In order to compute the electric conductivity, we follow the procedure in [28]. In this paper we work in the probe limit so that the fluctuation of the metric or the massive gauge field is ignored. For the conductivity only fluctuation $A_{x}(r)$ is relevant and let's work in the zero spatial momentum limit. Together with the background $\phi$ and the fluctuation $A_{x}$,

$$
A=\phi(r) d t+A_{x}(r) e^{-i \omega t} d x
$$

From the Maxwell equation,

$$
\frac{1}{\sqrt{-g}} \partial_{\mu}\left(\sqrt{-g} F^{\mu x}\right)=2 \psi^{2} A^{x}
$$

we find

$$
A_{x}^{\prime \prime}+\left[\frac{f^{\prime}(r)}{f(r)}-\frac{z-1}{r}\right] A_{x}^{\prime}+\left[\frac{\omega^{2} r^{2 z-2}}{f^{2}(r)}-\frac{2 \psi^{2}}{r^{2} f(r)}\right] A_{x}=0 .
$$

At the horizon we choose the infalling boundary condition,

$$
A_{x} \propto f(r)^{-i \omega r_{H}^{z} / 2}
$$

Near the boundary, the field behaves as

$$
A_{x}=A_{x(0)}+A_{x(1)} r^{z}+\ldots
$$

where $A_{x(0)}$ gives the background electric field in the dual field theory $E_{x}=i \omega A_{x(0)}$ and $A_{x(1)}$ is related to the expectation of electric current $J_{x}$. 

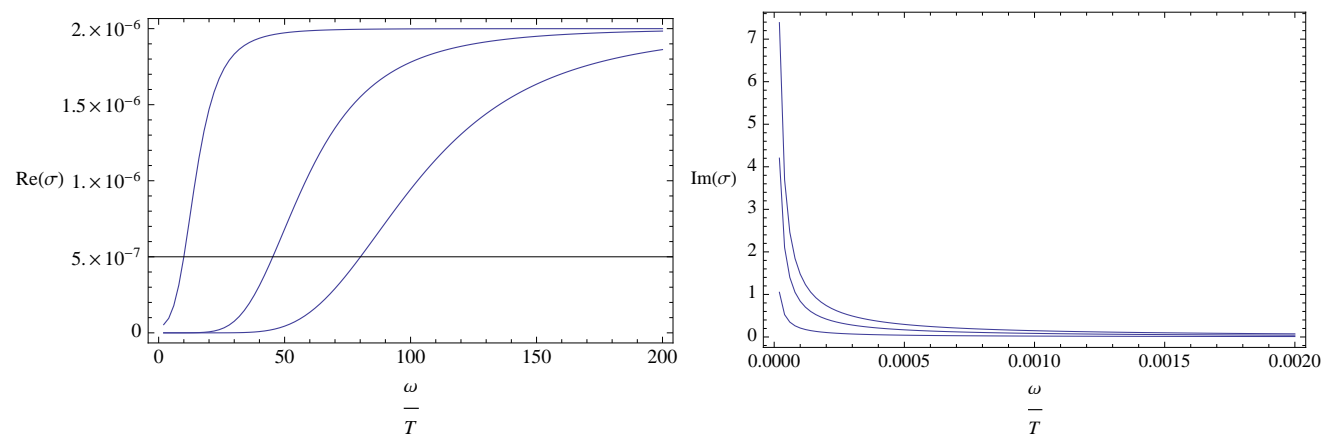

Figure 3: Conductivity at $T<T_{c}$. The temperature $T=0.512 T_{c}, T=0.155 T_{c}$, and $T=0.090 T_{c}$ from up to down in left figure and opposite in the right one.

For the gauge field (38), the Maxwell action reduces to

$$
S=-\frac{2}{4} \int d^{4} x \sqrt{-g}\left[g^{r r}\left(g^{x x} A_{x}^{2}+g^{t t} \phi^{2}\right)-g^{t t} g^{x x} \omega^{2} A_{x}^{2}\right]
$$

then the expectation value of the electric current can be obtained from this action,

$$
\left\langle J^{x}\right\rangle=\frac{\delta S_{\text {on-shell }}}{\delta A_{x(0)}}=-\lim _{r \rightarrow 0} \frac{\delta S}{\delta \partial_{r} A_{x}},
$$

with the notation $\partial_{r} A_{x}=A_{x}^{\prime}(r)$. Finally, we obtain

$$
\left\langle J^{x}\right\rangle=\lim _{r \rightarrow 0} \sqrt{-g} g^{r r}\left(g^{x x} A_{x}^{\prime}\right)=z A_{x(1)},
$$

which gives the conductivity

$$
\sigma(\omega)=\frac{\left\langle J^{x}\right\rangle}{E_{x}}=-\frac{i}{\omega} \frac{z A_{x(1)}}{A_{x(0)}} .
$$

All left is to solve equation (40) in order to obtain the electric conductivity in 46. In particular, we plot the conductivity at $T<T_{c}$. Near $\omega=0$, we observed a pole for the imaginary part. It means that DC conductivity becomes a delta function when condensation happens.

Apparently, there is a gap in Fig 3. However, as pointed out in [36], they may not be the genuine gap. One way to see this is to work out the the real part of the conductivity at low frequency at the zero temperature with full consideration of the backreaction to the gravity background. For charged Lifshitz solution (analogous to RN), we refer to [37]. Furthermore, the zero temperature limit from finite temperature is subtle, since a part of solutions at finite temperature depend on $\mathrm{T}$ only by the combination $\omega / T$. Therefore we can not take limit $\mathrm{T}$ goes to zero from the finite temperature. We postpone the examination of the zero temperature limit separately to future investigation. 


\section{Acknowledgements:}

We acknowledge Wei-Shui Xu, Li-Ming Cao, Yan Liu, Zhao-long Wang and Jian-Feng $\mathrm{Wu}$, Shuo Yang for useful discussions. Shan-Shan $\mathrm{Xu}$ would like to express thanks to J.X.Lu for his encouragement. Y.Zhou shall thank his advisor Miao Li for encouraging this work. The work of SJS was supported by KOSEF Grant R01-2007-000-10214-0 and also by NRF grant through CQUeST with grant number 2005-0049409.

\section{References}

[1] J. M. Maldacena, "The large N limit of superconformal field theories and supergravity," Adv. Theor. Math. Phys. 2 (1998) 231 [Int. J. Theor. Phys. 38 (1999) 1113] arXiv:hep-th/9711200.

[2] A. M. Polyakov, Int. J. Mod. Phys. A 17S1, 119 (2002) arXiv:hep-th/0110196.

[3] K. G. Wilson Phys. Rev. D10, 2445 (1974)

[4] G. Policastro, D. T. Son and A. O. Starinets, Phys. Rev. Lett. 87(2001) 081601, hep-th/0104066.

[5] S. J. Sin and I. Zahed, Phys. Lett. B608(2005)265, hep-th/0407215;

E. Shuryak, S-J. Sin and I. Zahed, "A Gravity Dual of RHIC Collisions," J.Korean Phys. Soc. 50, 384 (2007), hep-th/0511199.

[6] For recent review, see

D. Mateos, "String Theory and Quantum Chromodynamics," Class. Quant. Grav. 24, S713 (2007) arXiv:0709.1523 [hep-th]].

[7] S. A. Hartnoll and P. Kovtun, "Hall conductivity from dyonic black holes," Phys. Rev. D 76, 066001 (2007) arXiv:0704.1160 [hep-th]].

[8] S. A. Hartnoll, P. K. Kovtun, M. Muller and S. Sachdev, "Theory of the Nernst effect near quantum phase transitions in condensed matter, and in dyonic black holes," Phys. Rev. B 76, 144502 (2007) arXiv:0706.3215 [cond-mat.str-el]].

[9] S. A. Hartnoll and C. P. Herzog, "Ohm's Law at strong coupling: S duality and the cyclotron resonance," Phys. Rev. D 76, 106012 (2007) [arXiv:0706.3228 [hep-th]].

[10] S. A. Hartnoll and C. P. Herzog, "Impure AdS/CFT," arXiv:0801.1693 [hep-th]. 
[11] S. A. Hartnoll, C. P. Herzog and G. T. Horowitz, "Building a Holographic Superconductor," Phys. Rev. Lett. 101, 031601 (2008) [arXiv:0803.3295 [hep-th]].

[12] S. A. Hartnoll, C. P. Herzog and G. T. Horowitz, "Holographic Superconductors," JHEP 0812, 015 (2008) arXiv:0810.1563 [hep-th]].

[13] M. Ammon, J. Erdmenger, M. Kaminski and P. Kerner, "Flavor Superconductivity from Gauge/Gravity Duality," arXiv:0903.1864 [hep-th].

[14] G. T. Horowitz and M. M. Roberts, "Holographic Superconductors with Various Condensates," Phys. Rev. D 78, 126008 (2008) arXiv:0810.1077 [hep-th]].

[15] M. Fujita, W. Li, S. Ryu and T. Takayanagi, "Fractional Quantum Hall Effect via Holography: Chern-Simons, Edge States, arXiv:0901.0924 [hep-th].

[16] D. T. Son, "Toward an AdS/cold atoms correspondence: a geometric realization of the Schroedinger symmetry," Phys. Rev. D 78, 046003 (2008) arXiv:0804.3972 [hepth]]. A. Adams, K. Balasubramanian and J. McGreevy, "Hot Spacetimes for Cold Atoms," JHEP 0811 (2008) 059 [arXiv:0807.1111 [hep-th]]. C. P. Herzog, M. Rangamani and S. F. Ross, "Heating up Galilean holography," JHEP 0811, 080 (2008) arXiv:0807.1099 [hep-th]].

[17] S. Kachru, X. Liu and M. Mulligan, Phys. Rev. D 78, 106005 (2008) arXiv:0808.1725 [hep-th]].

[18] R. B. Mann, "Lifshitz Topological Black Holes," JHEP 0906, 075 (2009) arXiv:0905.1136 [hep-th]].

[19] G. Bertoldi, B. A. Burrington and A. Peet, "Black Holes in asymptotically Lifshitz spacetimes with arbitrary critical exponent," arXiv:0905.3183 [hep-th].

[20] G. Bertoldi, B. A. Burrington and A. W. Peet, "Thermodynamics of black branes in asymptotically Lifshitz spacetimes," arXiv:0907.4755 [hep-th].

[21] U. H. Danielsson and L. Thorlacius, "Black holes in asymptotically Lifshitz spacetime," JHEP 0903, 070 (2009) arXiv:0812.5088 [hep-th]].

[22] E. J. Brynjolfsson, U. H. Danielsson, L. Thorlacius and T. Zingg, "Holographic Superconductors with Lifshitz Scaling," arXiv:0908.2611 [hep-th].

[23] M. Taylor, "Non-relativistic holography," arXiv:0812.0530 [hep-th]. 
[24] K. Balasubramanian and J. McGreevy, "An analytic Lifshitz black hole," arXiv:0909.0263 [hep-th].

[25] E. Ayon-Beato, A. Garbarz, G. Giribet and M. Hassaine, "Lifshitz Black Hole in Three Dimensions," arXiv:0909.1347 [hep-th].

[26] R. G. Cai, Y. Liu and Y. W. Sun, "A Lifshitz Black Hole in Four Dimensional $R^{2}$ Gravity," arXiv:0909.2807 [hep-th].

[27] W. Li, T. Nishioka and T. Takayanagi, "Some No-go Theorems for String Duals of Non-relativistic Lifshitz-like Theories," arXiv:0908.0363 [hep-th].

[28] S. A. Hartnoll, "Lectures on holographic methods for condensed matter physics," arXiv:0903.3246 [hep-th].

[29] D. T. Son and A. O. Starinets, "Minkowski-space correlators in AdS/CFT correspondence: Recipe and applications," JHEP 0209, 042 (2002) arXiv:hep-th/0205051.

[30] S. S. Gubser, "Breaking an Abelian gauge symmetry near a black hole horizon," arXiv:0801.2977 [hep-th].

[31] N. Aizawa and V. K. Dobrev, arXiv:0906.0257 [hep-th].

[32] R. A. Konoplya and A. Zhidenko, arXiv:0909.2138 [hep-th].

[33] P. Koroteev and M. Libanov, JHEP 0802, 104 (2008) [arXiv:0712.1136 [hep-th]].

[34] S. Pu, S. J. Sin and Y. Zhou, arXiv:0903.4185 [hep-th].

[35] S. A. Hartnoll, J. Polchinski, E. Silverstein and D. Tong, JHEP 1004, 120 (2010) arXiv:0912.1061 [hep-th]].

[36] G. T. Horowitz and M. M. Roberts, JHEP 0911, 015 (2009) arXiv:0908.3677 [hepth]].

[37] E. J. Brynjolfsson, U. H. Danielsson, L. Thorlacius and T. Zingg, JHEP 1008, 027 (2010) [arXiv:1003.5361 [hep-th]]. E. J. Brynjolfsson, U. H. Danielsson, L. Thorlacius and T. Zingg, arXiv:1004.5566 [hep-th]. 